\title{
A Characterization of Jordan Homomorphism on Banach Algebras
}

\begin{abstract}
Abbas Zivari-Kazempour
Department of Mathematics, Ayatollah Borujerdi University, Borujerd, Iran

Correspondence should be addressed to Abbas Zivari-Kazempour; zivari@abru.ac.ir

Received 20 October 2013; Accepted 17 December 2013; Published 29 January 2014

Academic Editors: C.-J. Zhao and Q. Zhu

Copyright ( 2014 Abbas Zivari-Kazempour. This is an open access article distributed under the Creative Commons Attribution License, which permits unrestricted use, distribution, and reproduction in any medium, provided the original work is properly cited.
\end{abstract}

Let $\mathscr{A}$ and $\mathscr{B}$ be Banach algebras and let $\varphi: \mathscr{A} \rightarrow \mathscr{B}$ be a Jordan homomorphism. We show that, under special hypotheses, $\varphi$ is ring homomorphism. Some related results are given as well.

\section{Introduction}

Let $\mathscr{A}$ and $\mathscr{B}$ be Banach algebras and let $\varphi: \mathscr{A} \rightarrow \mathscr{B}$ be a linear map. Then $\varphi$ is called Jordan homomorphism if

$$
\varphi(a b+b a)=\varphi(a) \varphi(b)+\varphi(b) \varphi(a), \quad(a, b \in \mathscr{A}),
$$

or equivalently, $\varphi\left(a^{2}\right)=\varphi(a)^{2}$ for all $a \in \mathscr{A}$. Moreover, if $\varphi$ is multiplicative, that is,

$$
\varphi(a b)=\varphi(a) \varphi(b), \quad(a, b \in \mathscr{A}),
$$

then $\varphi$ is called ring homomorphism. It is obvious that ring homomorphisms are Jordan, but the converse is false, in general. In fact, the converse is true under certain conditions. For example, each Jordan homomorphism from a commutative Banach algebra $\mathscr{A}$ into $\mathbb{C}$ is a ring homomorphism.

In [1], Żelazko proved that each Jordan homomorphism of Banach algebra $\mathscr{A}$ into a semisimple commutative Banach algebra $\mathscr{B}$ is ring homomorphism (see also Theorem 1.1 of [2]).

In [3], Eshaghi Gordji studied the concept of $n$-Jordan homomorphisms for complex algebras. The linear map $\varphi$ : $\mathscr{A} \rightarrow \mathscr{B}$ is said to be $n$-Jordan homomorphism if, for all $a \in \mathscr{A}, \varphi\left(a^{n}\right)=\varphi(a)^{n}$. A 2-Jordan homomorphism is a Jordan homomorphism, in the usual sense.

He proved that each 3-Jordan homomorphism between commutative algebras $\mathscr{A}$ and $\mathscr{B}$ is 3-ring homomorphism [3] .
Moreover, he showed that Zelazko's theorem is valid for 3Jordan homomorphism.

Throughout the paper, all Banach algebras are assumed to be over the complex field $\mathbb{C}$.

The proof of the following lemma is contained in [4].

Lemma 1. Let $\mathscr{A}$ and $\mathscr{B}$ be Banach algebras and let $\varphi: \mathscr{A} \rightarrow$ $\mathscr{B}$ be a Jordan homomorphism. Then for all $a, b \in \mathscr{A}$,

$$
\varphi(a b a)=\varphi(a) \varphi(b) \varphi(a) .
$$

\section{Jordan Homomorphism}

In general, the kernel of an $n$-Jordan homomorphism may not be an ideal. For example, let $\mathscr{A}$ be the algebra of all $3 \times 3$ matrices having 0 on and below the diagonal. In this algebra product of any 3 elements is equal to 0 , so any linear map from $\mathscr{A}$ into itself is a 3 -Jordan homomorphism but its kernel does not need to be an ideal (see also [3], for the case $n=4$ ).

For Jordan homomorphism, we have the following.

Proposition 2. Let $\varphi$ be a Jordan homomorphism from Banach algebra $\mathscr{A}$ into a semiprime commutative Banach algebra $\mathscr{B}$. Then $\operatorname{ker} \varphi$ is an ideal of $\mathscr{A}$.

Proof. Let $a \in \operatorname{ker} \varphi$ and $x \in \mathscr{A}$ be arbitrary. Since $\varphi$ is Jordan and $\varphi(a)=0$, we have

$$
\varphi(a x+x a)=\varphi(a) \varphi(x)+\varphi(x) \varphi(a)=0 .
$$


It follows that $\varphi(x a)=-\varphi(a x)$. Put $b=x a x$, and then

$$
\begin{aligned}
0=\varphi(b a+a b) & =\varphi(x a x a)+\varphi(\text { axax }) \\
& =\varphi\left((x a)^{2}\right)+\varphi\left((a x)^{2}\right) \\
& =\varphi(x a)^{2}+\varphi(a x)^{2} \\
& =(-\varphi(a x))^{2}+\varphi(a x)^{2} \\
& =2 \varphi(a x)^{2} .
\end{aligned}
$$

Hence, $\varphi(a x)$ is a central nilpotent element of $\mathscr{B}$, but semiprime algebra contains not of central nilpotent non-zero elements; therefore, $\varphi(a x)=0$. Thus, $a x \in \operatorname{ker} \varphi$. Similarly, $x a \in \operatorname{ker} \varphi$.

Theorem 3. Let $\varphi$ be a Jordan homomorphism of Banach algebra $\mathscr{A}$ into a semiprime commutative Banach algebra $\mathscr{B}$. If there exist linear functional $f$ on $\mathscr{A}$ such that $\operatorname{ker} f \subseteq \operatorname{ker} \varphi$, then $\varphi$ is ring homomorphism.

Proof. Let $a, b$ be arbitrary elements of $\mathscr{A}$. Then the result is clear, if $\varphi(a)$ or $\varphi(b)$ is zero. We first assume that $\varphi(a)=\varphi(b)$. Then $a-b \in \operatorname{ker} \varphi$, and so by the above proposition $(a-b) b \in$ $\operatorname{ker} \varphi$, it follows that

$$
\varphi(a b)=\varphi\left(b^{2}\right)=\varphi(b)^{2}=\varphi(a) \varphi(b) .
$$

Therefore, $\varphi$ is ring homomorphism. Now suppose that $\varphi(a) \neq \varphi(b)$, and both of them be nonzero. Take $\alpha=1 / f(a)$, $\beta=1 / f(b), x=\alpha a$, and $y=\beta b$. Then $f(x)=f(y)$, so

$$
x-y \in \operatorname{ker} f \subseteq \operatorname{ker} \varphi .
$$

Hence, $\varphi(x)=\varphi(y)$, and the above argument shows that

$$
\varphi(x y)=\varphi(x) \varphi(y),
$$

which implies that

$$
\varphi(a b)=\frac{1}{\alpha \beta} \varphi(x y)=\frac{1}{\alpha \beta} \varphi(x) \varphi(x)=\varphi(a) \varphi(b) .
$$

This completes the proof.

For certain calculations, we use the following notation which is called the Lie product of $a$ and $b$ :

$$
[a, b]=a b-b a, \quad(a, b \in \mathscr{A}) .
$$

Theorem 4. Let $\varphi$ be a Jordan homomorphism from a commutative Banach algebra $\mathscr{A}$, with its range dense into a semiprime Banach algebra $\mathscr{B}$. Then $\varphi$ is ring homomorphism.

Proof. By Lemma 6.3.2(e) of $[4]$, we have $\varphi\left([a, b]^{2}\right)=[\varphi(a)$, $\varphi(b)]^{2}$ for all $a, b \in \mathscr{A}$. Since $\mathscr{A}$ is commutative, $[a, b]=0$, so

$$
[\varphi(a), \varphi(b)]^{2}=0
$$

The continuity of Lie product and density of the range of $\varphi$ imply that $[\varphi(a), \varphi(b)]$ is a central nilpotent element of $\mathscr{B}$ and so it must be zero. Therefore,

$$
\varphi(a) \varphi(b)=\varphi(b) \varphi(a) .
$$

Thus, if $a b=b a$, then we conclude that

$$
\begin{aligned}
\varphi(a b) & =\frac{1}{2} \varphi(a b+b a) \\
& =\frac{1}{2}(\varphi(a) \varphi(b)+\varphi(b) \varphi(a)) \\
& =\varphi(a) \varphi(b) .
\end{aligned}
$$

Therefore, $\varphi$ is ring homomorphism.

As a consequence, we deduce the following result.

Corollary 5. Let $\varphi$ be a Jordan homomorphism from a Banach algebra $\mathscr{A}$, with its range dense into a semiprime Banach algebra $\mathscr{B}$. If $\mathscr{A}$ is commutative, then so is $\mathscr{B}$.

Theorem 6. Let $\varphi$ be a Jordan homomorphism of Banach algebra $\mathscr{A}$ into a unital Banach algebra $\mathscr{B}$ with unit element $e$. Then, for all $a \in \mathscr{A}$ with $\|a\|<1, \varphi(a) \neq e$.

Proof. Suppose there exist $a \in \mathscr{A}$ with $\|a\|<1$ and $\varphi(a)=e$. Take $b=\sum_{n=1}^{\infty} a^{n}$. Then $a b+a=b$, so

$$
b a b+b a=b^{2}, \quad a b a+a^{2}=b a .
$$

Thus, by Lemma 1 we have

$$
\begin{aligned}
& \varphi(b) \varphi(a) \varphi(b)+\varphi(b a)=\varphi\left(b^{2}\right) \\
& \varphi(a) \varphi(b) \varphi(a)+\varphi\left(a^{2}\right)=\varphi(b a)
\end{aligned}
$$

Since $\varphi$ is Jordan and $\varphi(a)=e$, (15) implies that $\varphi(b a)=0$ and so $\varphi(b)=-e$, by (16). Therefore,

$$
\begin{aligned}
0 & =\varphi(a b) \varphi(b a)+\varphi(b a) \varphi(a b) \\
& =\varphi(a b b a+b a a b) \\
& =\varphi\left(a b^{2} a+b a^{2} b\right) \\
& =\varphi(a) \varphi\left(b^{2}\right) \varphi(a)+\varphi(b) \varphi\left(a^{2}\right) \varphi(b) \\
& =2 e .
\end{aligned}
$$

It follows that $2 e=0$, which is a contradiction. This completes the proof.

Let $\mathscr{A}$ be a Banach algebra. It is well known that, on the second dual space $\mathscr{A}^{\prime \prime}$ of $\mathscr{A}$, there are two multiplications, called the first and second Arens products which make $\mathscr{A}^{\prime \prime}$ into a Banach algebra (see $[5,6])$. By definition, the first Arens product $\square$ on $\mathscr{A}^{\prime \prime}$ is induced by the left $\mathscr{A}$-module structure on $\mathscr{A}$. That is, for each $\Phi, \Psi \in \mathscr{A}^{\prime \prime}, f \in \mathscr{A}^{\prime}$, and $a, b \in \mathscr{A}$, we have

$$
\begin{gathered}
\langle\Phi \square \Psi, f\rangle=\langle\Phi, \Psi \cdot f\rangle, \quad\langle\Psi \cdot f, a\rangle=\langle\Psi, f \cdot a\rangle, \\
\langle f \cdot a, b\rangle=\langle f, a b\rangle .
\end{gathered}
$$


Similarly, the second Arens product $\diamond$ on $\mathscr{A}^{\prime \prime}$ is defined by considering $\mathscr{A}$ as a right $\mathscr{A}$-module.

An element $\Phi_{0} \in \mathscr{A}^{\prime \prime}$ is called mixed unit if it is a right unit for $\left(\mathscr{A}^{\prime \prime}, \square\right)$ and a left unit for $\left(\mathscr{A}^{\prime \prime}, \diamond\right)$. It is well known that an element $\Phi_{0} \in \mathscr{A}^{\prime \prime}$ is a mixed unit if and only if it is a weak ${ }^{*}$ cluster point of some bounded approximate identity $\left(e_{\alpha}\right)_{\alpha \in I}$ in $\mathscr{A}[7]$

Theorem 7. Let $\mathscr{A}$ and $\mathscr{B}$ be Banach algebras and let $\varphi$ : $\mathscr{A} \rightarrow \mathscr{B}$ be a Jordan homomorphism. Then $\varphi^{\prime \prime}$ is a Jordan homomorphism.

Proof. Let $a \in \mathscr{A}$ and $f \in \mathscr{B}^{\prime}$; then we have

$$
\begin{aligned}
\left\langle\varphi^{\prime}(f) \cdot a, a\right\rangle & =\left\langle f, \varphi\left(a^{2}\right)\right\rangle=\langle f, \varphi(a) \varphi(a)\rangle \\
& =\langle f \cdot \varphi(a), \varphi(a)\rangle=\left\langle\varphi^{\prime}(f \cdot \varphi(a)), a\right\rangle
\end{aligned}
$$

It follows that $\varphi^{\prime}(f) \cdot a=\varphi^{\prime}(f \cdot \varphi(a))$ and thus for all $\Phi \in \mathscr{A}^{\prime \prime}$,

$$
\begin{aligned}
\left\langle\Phi \cdot \varphi^{\prime}(f), a\right\rangle & =\left\langle\Phi, \varphi^{\prime}(f) \cdot a\right\rangle \\
& =\left\langle\Phi, \varphi^{\prime}(f \cdot \varphi(a))\right\rangle \\
& =\left\langle\varphi^{\prime \prime}(\Phi), f \cdot \varphi(a)\right\rangle \\
& =\left\langle\varphi^{\prime \prime}(\Phi) \cdot f, \varphi(a)\right\rangle \\
& =\left\langle\varphi^{\prime}\left(\varphi^{\prime \prime}(\Phi) \cdot f\right), a\right\rangle .
\end{aligned}
$$

Therefore, $\Phi \cdot \varphi^{\prime}(f)=\varphi^{\prime}\left(\varphi^{\prime \prime}(\Phi) \cdot f\right)$. Now we have

$$
\begin{aligned}
\left\langle\varphi^{\prime \prime}\left(\Phi^{2}\right), f\right\rangle & =\left\langle\Phi^{2} \circ \varphi^{\prime}, f\right\rangle \\
& =\left\langle\Phi, \Phi \cdot \varphi^{\prime}(f)\right\rangle \\
& =\left\langle\Phi, \varphi^{\prime}\left(\varphi^{\prime \prime}(\Phi) \cdot f\right)\right\rangle \\
& =\left\langle\varphi^{\prime \prime}(\Phi), \varphi^{\prime \prime}(\Phi) \cdot f\right\rangle \\
& =\left\langle\varphi^{\prime \prime}(\Phi)^{2}, f\right\rangle .
\end{aligned}
$$

Therefore, $\varphi^{\prime \prime}\left(\Phi^{2}\right)=\varphi^{\prime \prime}(\Phi)^{2}$, as required.

The proof of the next result is the same as that of the above theorem.

Theorem 8. Let $\mathscr{A}$ and $\mathscr{B}$ be Banach algebras and let $\varphi$ : $\mathscr{A} \rightarrow \mathscr{B}$ be a 3-Jordan homomorphism. Then $\varphi^{\prime \prime}$ is a 3-Jordan homomorphism.

Corollary 9. Suppose $\varphi: \mathscr{A} \rightarrow \mathscr{B}$ is a 3-Jordan homomorphism between Banach algebras. If $\mathscr{B}^{\prime \prime}$ is a commutative and semisimple, then $\varphi^{\prime \prime}$ is 3-ring homomorphism.

Proof. It follows of above Theorem and Theorem 2.5 of [3].
Proposition 10. Let $\varphi: \mathscr{A} \rightarrow \mathscr{B}$ be a 3-Jordan homomorphism between commutative Banach algebras. If $\mathscr{A}$ has a BAI, then $h: \mathscr{A}^{\prime \prime} \rightarrow \mathscr{B}^{\prime \prime}$ defined by

$$
h(\Phi):=\varphi^{\prime \prime}\left(\Phi_{0}\right) \square \varphi^{\prime \prime}(\Phi), \quad\left(\Phi \in \mathscr{A}^{\prime \prime}\right),
$$

is a homomorphism.

Proof. This follows of Theorem 2.2 of [3] and Theorem 3.2 of [8].

We recall that an element $a$ of $C^{*}$-algebra $\mathscr{A}$ is said to be partial isometry if $a a^{*} a=a$.

Theorem 11. Let $\mathscr{A}$ and $\mathscr{B}$ be $C^{*}$-algebras and let $\varphi: \mathscr{A} \rightarrow \mathscr{B}$ be a Jordan homomorphism preserving the involution. If $\varphi$ is weakly norm continuous, then $\|\varphi\| \leq 1$.

Proof. By Lemma 1, for all $a \in \mathscr{A}$ we have

$$
\varphi\left(a a^{*} a\right)=\varphi(a) \varphi(a)^{*} \varphi(a) .
$$

Thus, $\varphi$ preserves the partial isometries. Since every partial isometry $a$ has norm $\|a\| \leq 1$, hence for partial isometries $a_{1}, \ldots, a_{n}$ and positive scalers $\alpha_{1}, \ldots, \alpha_{n}$ with $\alpha_{1}+\cdots+\alpha_{n}=1$, we conclude that

$$
\left\|\varphi\left(\sum_{i=1}^{n} \alpha_{i} a_{i}\right)\right\|=\left\|\sum_{i=1}^{n} \alpha_{i} \varphi\left(a_{i}\right)\right\| \leq \sum_{i=1}^{n} \alpha_{i}\left\|\varphi\left(a_{i}\right)\right\| \leq 1 .
$$

By assumption $\varphi$ is weakly norm continuous, so it follows that $\|\varphi\| \leq 1$.

\section{Conflict of Interests}

The author declares that there is no conflict of interests regarding the publication of this paper.

\section{References}

[1] W. Żelazko, "A characterization of multiplicative linear functionals in complex Banach algebras," Studia Mathematica, vol. 30, pp. 83-85, 1968.

[2] T. Miura, S.-E. Takahasi, and G. Hirasawa, "Hyers-UlamRassias stability of Jordan homomorphisms on Banach algebras," Journal of Inequalities and Applications, vol. 2005, no. 4, pp. 435-441, 2005.

[3] M. Eshaghi Gordji, " $n$-Jordan homomorphisms," Bulletin of the Australian Mathematical Society, vol. 80, no. 1, pp. 159-164, 2009.

[4] T. Palmer, Banach Algebras and the General Theory C*-Algebras, vol. 1, Cambridge University Press, Cambridge, UK, 1994.

[5] H. G. Dales, Banach Algebras and Automatic Continuity, vol. 24 of London Mathematical Society Monographs, Clarendon Press, Oxford, UK, 2000.

[6] H. G. Dales and A. T.-M. Lau, "The second duals of beurling algebras," Memoirs of the American Mathematical Society, vol. 177, no. 836, 2005.

[7] F. F. Bonsall and J. Duncan, Complete Normed Algebra, Springer, New York, NY, USA, 1973.

[8] J. Bračič and M. S. Moslehian, "On automatic continuity of 3homomorphisms on Banach algebras," Bulletin of the Malaysian Mathematical Sciences Society, vol. 30, no. 2, pp. 195-200, 2007. 


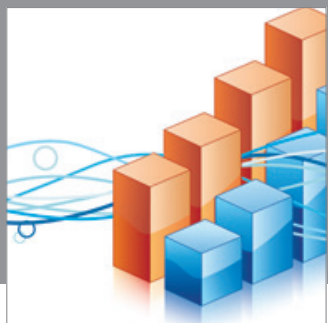

Advances in

Operations Research

mansans

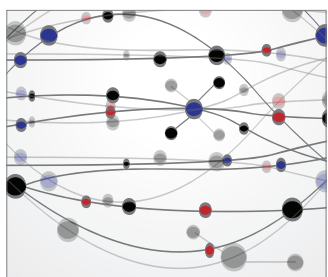

The Scientific World Journal
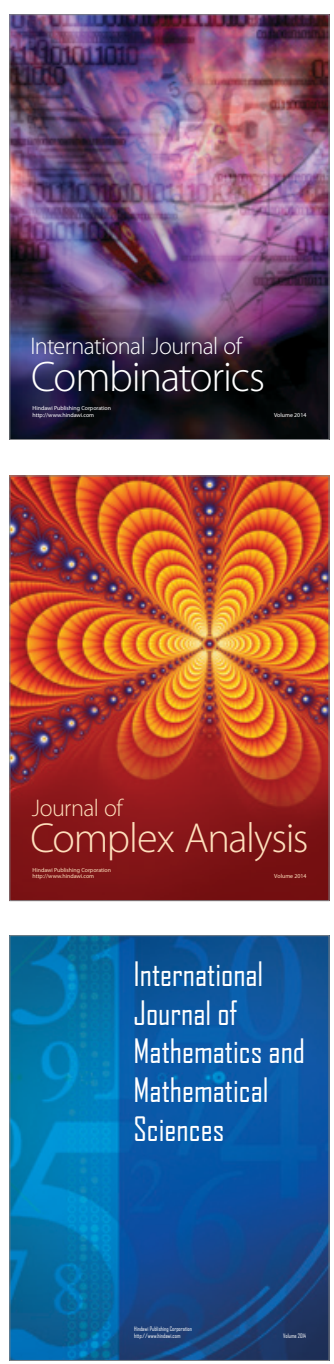
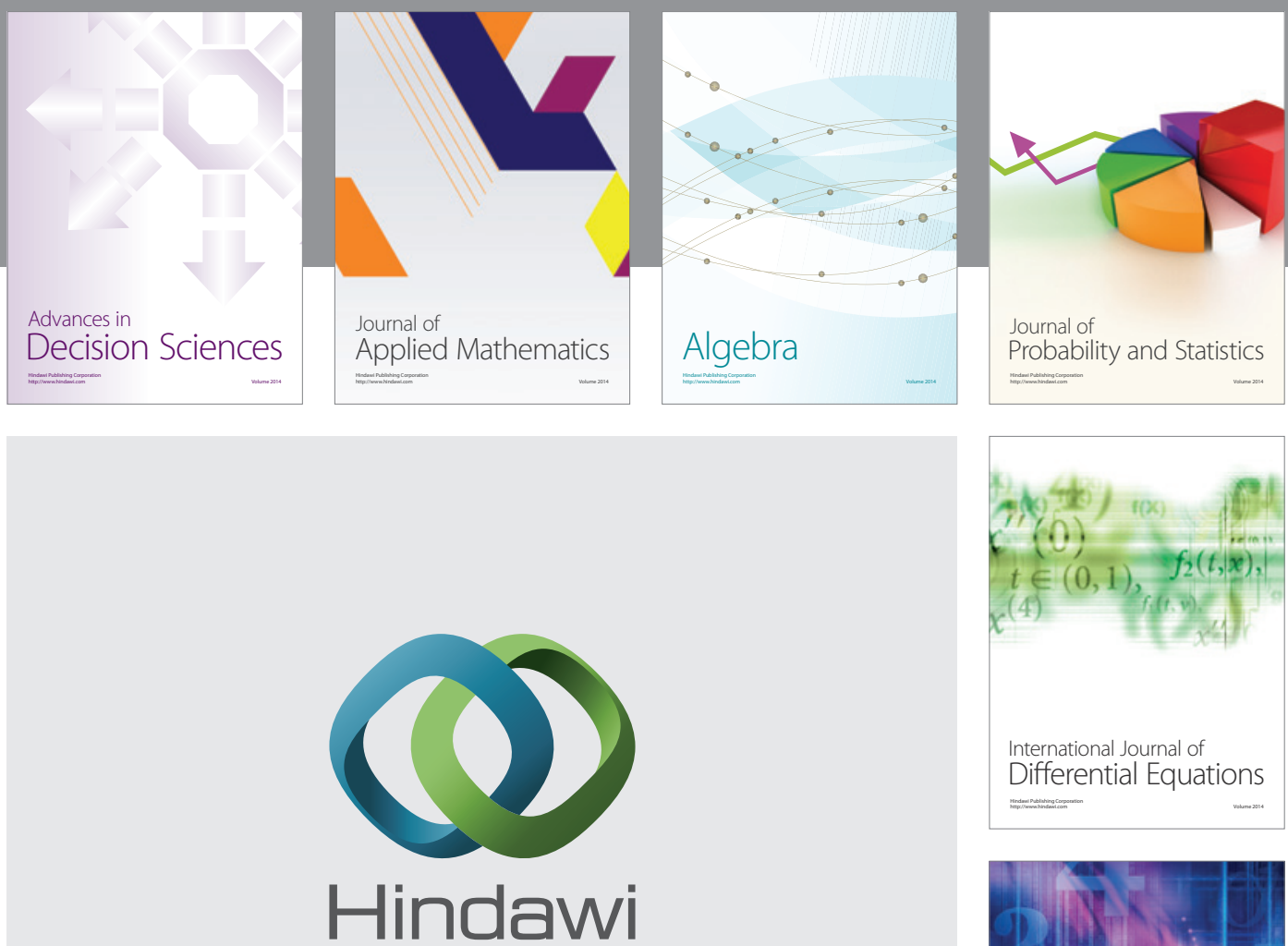

Submit your manuscripts at http://www.hindawi.com
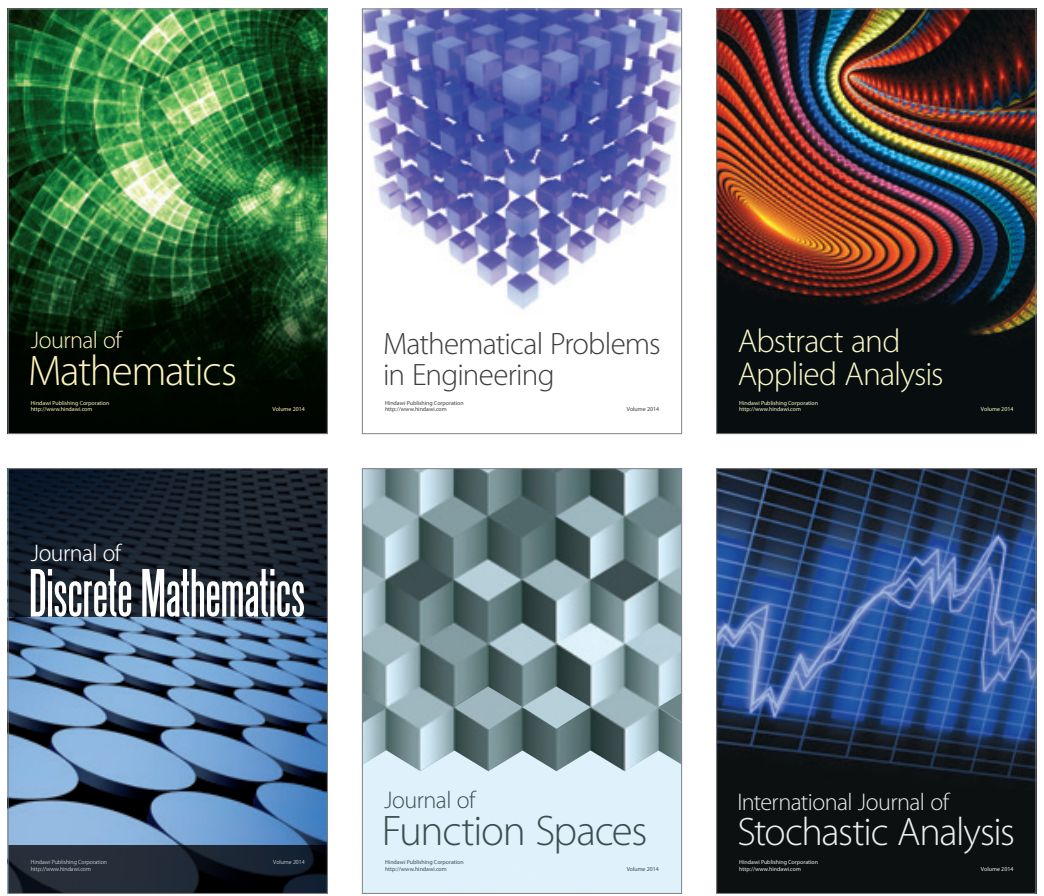

Journal of

Function Spaces

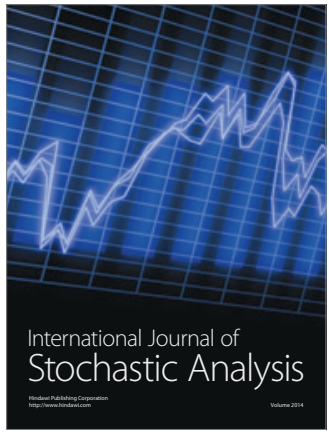

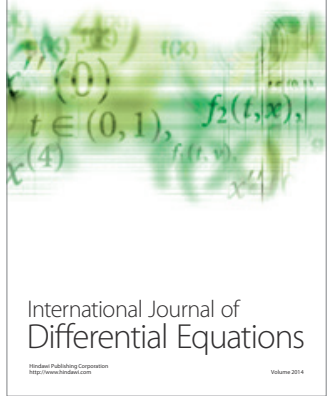
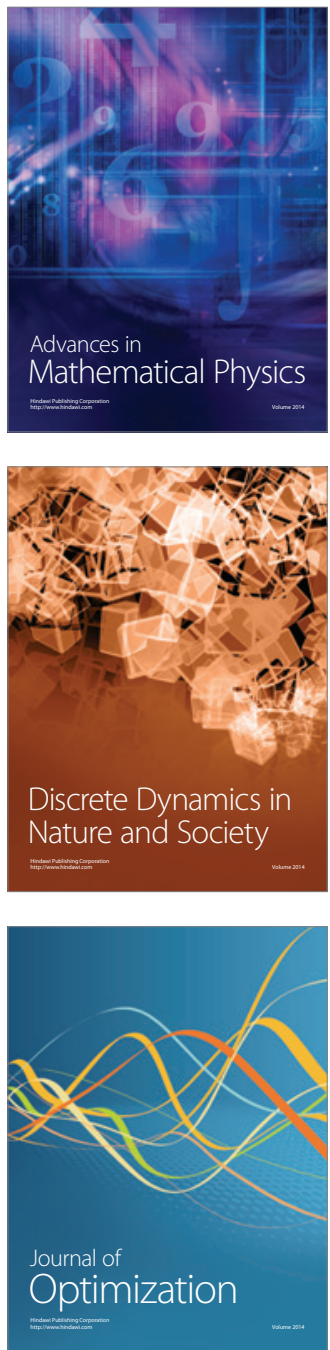